\title{
Shift in VEGFA isoform balance towards more angiogenic variants is associated with tumor stage and differentiation of human hepatocellular carcinoma
}

\author{
Mikhail S Chesnokov ${ }^{1}$, Polina A Khesina ${ }^{1,2}$, Darya A Shavochkina ${ }^{1}$, Inna F Kustova ${ }^{1}$, Leonid M Dyakov ${ }^{1}$, \\ Olga V Morozova ${ }^{1}$, Nikolai S Mugue ${ }^{3}$, Nikolay E Kudashkin ${ }^{4}$, Ekaterina A Moroz ${ }^{4}$, Yuri I Patyutko ${ }^{4}$, \\ Natalia L Lazarevich ${ }^{\text {Corresp. 1, } 2}$ \\ 1 Institute of Carcinogenesis, FSBI "N.N. Blokhin National Medical Research Center of Oncology" of the Ministry of Health of the Russian Federation, \\ Moscow, Russian Federation \\ 2 Biological Faculty, M.V. Lomonosov Moscow State University, Moscow, Russian Federation \\ 3 N.K. Koltzov Institute of Developmental Biology of Russian Academy of Sciences, Moscow, Russian Federation \\ 4 Institute of Clinical Oncology, FSBI "N.N. Blokhin National Medical Research Center of Oncology" of the Ministry of Health of the Russian Federation, \\ Moscow, Russian Federation \\ Corresponding Author: Natalia L Lazarevich \\ Email address: lazarevich.nl@gmail.com
}

Background. Hepatocellular carcinoma (HCC) is the most common and aggressive type of malignant liver tumors. HCC progression depends significantly on its vascularization and formation of new blood vessels. Vascular endothelial growth factor A (VEGFA) is a crucial regulator of tumor vascularization and components of VEGF-induced cell signaling pathways are important targets of therapeutical drugs that demonstrated the highest efficiency in case of advanced HCC (sorafenib and regorafenib). VEGFA is expressed as a set of isoforms with different functional properties thus VEGFA isoform expression pattern may affect tumor sensitivity to anti-angiogenic drugs. However, information about VEGFA isoforms expression in HCC is still incomplete and contradictory. Present paper aims to quantitatively investigate VEGFA isoform expression aberrations in HCC tissue.

Methods. 50 pairs of HCC and non-tumor tissue samples were used to evaluate VEGFA isoform spectrum using RT-PCR and quantitatively estimate changes in isoform expression using RT-qPCR. Correlations between these changes and tumor clinicopathological characteristics were analyzed.

Results. We identified VEGFA-189, VEGFA-165 and VEGFA-121 as predominant isoforms in liver tissue. Anti-angiogenic VEGFA-Xxxb variants constituted no more than $5 \%$ of all mature VEGFA transcripts detected and their expression was not changed significantly in HCC tissue. We demonstrated for the first time that the least active variant VEGFA-189 is frequently repressed in HCC $(p<0.001)$, while no uniform changes were detected for potent angiogenesis stimulators VEGFA-165 and VEGFA-121. Isoform balance in HCC shifts from VEGFA-189 towards VEGFA-165 or VEGFA-121 in majority of cases $(p<0.001)$. Changes in fractions, but not expression levels, of VEGFA-189 (decrease) and VEGFA-121 (increase) correlated with advanced TNM and BCLC tumor stages $(p<0.05)$, VEGFA-189 fraction reduction was also associated with poor tumor differentiation $(p<0.05)$.

Discussion. A distinct shift in VEGFA isoform balance towards more pro-angiogenic variants occurs in HCC tissue and may modulate overall impact of VEGFA signaling. We suppose that ratio between VEGFA isoforms is an important parameter governing HCC angiogenesis that may affect HCC progression and be used for optimizing the strategy of HCC therapy by predicting the response to anti-angiogenic drugs. 
1 Shift in VEGFA isoform balance towards more angiogenic variants is associated with

2 tumor stage and differentiation of human hepatocellular carcinoma

4 Mikhail S Chesnokov', Polina A Khesina ${ }^{1,2}$, Darya A Shavochkina ${ }^{1}$, Inna F Kustova ${ }^{1}$,

5 Leonid M Dyakov', Olga V Morozova ${ }^{1}$, Nikolai S Mugue³, Nikolay E Kudashkin ${ }^{4}$,

6 Ekaterina A Moroz ${ }^{4}$, Yuri I Patyutko ${ }^{4}$, Natalia L Lazarevich ${ }^{1,2}$

7

$8{ }^{1}$ Institute of Carcinogenesis, FSBI "N.N. Blokhin National Medical Research Center of

9 Oncology" of the Ministry of Health of the Russian Federation, 115478, Kashirskoye shosse 24,

10 Moscow, Russian Federation.

11 2Biological Faculty, M.V. Lomonosov Moscow State University, 119992, Leninskye gory 1,

12 Moscow, Russian Federation.

$13{ }^{3}$ N.K. Koltzov Institute of Developmental Biology of Russian Academy of Sciences , 119334,

14 Vavilova st. 26, Moscow, Russian Federation.

$15{ }^{4}$ Institute of Clinical Oncology, FSBI "N.N. Blokhin National Medical Research Center of

16 Oncology" of the Ministry of Health of the Russian Federation, 115478, Kashirskoye shosse 23,

17 Moscow, Russian Federation.

19 Corresponding Author:

20 Natalia Lazarevich

21 Phone: $+7(499) 3235655$

22 E-mail: lazarevich.nl@gmail.com 


\section{Abstract.}

25

26 Background. Hepatocellular carcinoma (HCC) is the most common and aggressive type of

27 malignant liver tumors. HCC progression depends significantly on its vascularization and

28 formation of new blood vessels. Vascular endothelial growth factor A (VEGFA) is a crucial

29 regulator of tumor vascularization and components of VEGF-induced cell signaling pathways are

30 important targets of therapeutical drugs that demonstrated the highest efficiency in case of

31 advanced HCC (sorafenib and regorafenib). VEGFA is expressed as a set of isoforms with

32 different functional properties thus VEGFA isoform expression pattern may affect tumor

33 sensitivity to anti-angiogenic drugs. However, information about VEGFA isoforms expression in

$34 \mathrm{HCC}$ is still incomplete and contradictory. Present paper aims to quantitatively investigate

35 VEGFA isoform expression aberrations in HCC tissue.

36 Methods. 50 pairs of HCC and non-tumor tissue samples were used to evaluate VEGFA isoform

37 spectrum using RT-PCR and quantitatively estimate changes in isoform expression using RT-

38 qPCR. Correlations between these changes and tumor clinicopathological characteristics were

39 analyzed.

40 Results. We identified VEGFA-189, VEGFA-165 and VEGFA-121 as predominant isoforms in

41 liver tissue. Anti-angiogenic VEGFA-xxxb variants constituted no more than 5\% of all mature

42 VEGFA transcripts detected and their expression was not changed significantly in HCC tissue.

43 We demonstrated for the first time that the least active variant VEGFA-189 is frequently

44 repressed in $\mathrm{HCC}(\mathrm{p}<0.001)$, while no uniform changes were detected for potent angiogenesis

45 stimulators VEGFA-165 and VEGFA-121. Isoform balance in HCC shifts from VEGFA-189

46 towards VEGFA-165 or VEGFA-121 in majority of cases $(\mathrm{p}<0.001)$. Changes in fractions, but 
47 not expression levels, of VEGFA-189 (decrease) and VEGFA-121 (increase) correlated with

48 advanced TNM and BCLC tumor stages $(\mathrm{p}<0.05)$, VEGFA-189 fraction reduction was also

49 associated with poor tumor differentiation $(\mathrm{p}<0.05)$.

50 Discussion. A distinct shift in VEGFA isoform balance towards more pro-angiogenic variants

51 occurs in HCC tissue and may modulate overall impact of VEGFA signaling. We suppose that

52 ratio between VEGFA isoforms is an important parameter governing HCC angiogenesis that may

53 affect HCC progression and be used for optimizing the strategy of HCC therapy by predicting

54 the response to anti-angiogenic drugs.

55

\section{Introduction.}

Hepatocellular carcinoma (HCC) is the most common and aggressive form of primary liver tumors that ranks the second place in cancer-related mortality rates (Llovet et al., 2016). Most HCC patients are diagnosed at advanced stages when the efficacy of existing therapeutic approaches is low and overall prognosis is poor (Wörns \& Galle, 2010). Identification of specific molecular markers and regulatory mechanisms underlying HCC development is of great clinical importance as it can result in development of novel drugs and strategies for targeted HCC therapy.

Angiogenesis is one of the most critical processes involved in HCC pathogenesis (Liu et al., 2017). It is mostly stimulated via vascular endothelial growth factor (VEGF) signaling pathway activated by secreted VEGF proteins interacting with membrane tyrosine-kinase receptors KDR and FLT-1 (Ferrara, Gerber \& LeCouter, 2003; Shen, Hsu \& Cheng, 2010). VEGF family includes 5 factors, of which VEGFA is the main driver of angiogenesis (Ferrara, 
70 Gerber \& LeCouter, 2003; Rapisarda \& Melillo, 2012; Vempati, Popel \& Mac Gabhann, 2014).

71 Multiple VEGFA isoforms generated through alternative splicing divide into two functional

72 groups. VEGFA-xxxa group (where " $\mathrm{xxx}$ " is protein chain length) is pro-angiogenic with

73 VEGFA-121, VEGFA-165 and VEGFA-189 isoforms being most common; these isoforms differ

74 by presence or absence of exons 6 and 7 (Fig. 1A) (Ferrara, Gerber \& LeCouter, 2003; Vempati,

75 Popel \& Mac Gabhann, 2014). VEGFA-xxxb isoforms, discerned by lack of exon 8a, act as

76 angiogenesis suppressors; the most common variant is VEGFA-165b (Fig. 1A) (Harper \& Bates,

77 2008). Since exons 6 and 7 encode heparin-binding domains (HBD) responsible for interaction

78 with extracellular matrix proteins, HBD-containing isoforms like VEGFA-189 are tightly bound

79 to the cell surface. VEGFA-165 is semi-soluble while VEGFA-121 is completely diffusible

80 (Ferrara, Gerber \& LeCouter, 2003; Vempati, Popel \& Mac Gabhann, 2014). Functional impact

81 of different VEGFA isoforms is also defined by receptors they interact with. VEGFA-165 is

82 more potent angiogenesis stimulator than VEGFA-121 and VEGFA-189; it acts mainly through

83 KDR-neuropilin-1 complex, which is the primary mediator of VEGF signaling (Ferrara, Gerber

84 \& LeCouter, 2003). Neuropilin-1 potentiates interactions between KDR and VEGFA-165, but

85 not VEGFA-121 and VEGFA-189, so they act through much weaker FLT-1 receptor (Rapisarda

86 \& Melillo, 2012; Vempati, Popel \& Mac Gabhann, 2014). VEGFA-189 is supposedly the

87 weakest angiogenesis stimulator among three isoforms due to its low solubility and necessity of

88 proteolytic processing (Plouët et al., 1997; Vempati, Popel \& Mac Gabhann, 2014).

89 High levels of VEGFA expression were reported in different types of malignant tumors

90 including $\mathrm{HCC}$ and are associated with advanced stages of disease, poor survival of patients and

91 high recurrence rate (Ferrara, Gerber \& LeCouter, 2003; Tseng et al., 2008; Shen, Hsu \& Cheng,

92 2010; Chekhonin et al., 2013). A number of VEGFA pathway inhibitors are currently under 
93 investigation or already approved for clinical use like bevacizumab, sunitinib, sorafenib and

94 regorafenib, the last two being shown the most efficient drugs for therapy of advanced HCC

95 (Shen, Hsu \& Cheng, 2010; Rapisarda \& Melillo, 2012; Bruix et al., 2017). However, little is

96 known about functional impact of alternatively spliced forms of VEGFA in

97 hepatocarcinogenesis, while changes in isoform expression pattern may have a considerable

98 effect upon tumor development (Ladomery, 2013; Berasain et al., 2014; Elizalde et al., 2014).

99 The importance of evaluation of VEGFA variants expression in tumors is clearly demonstrated

100 by investigation of clinical impact of anti-angiogenic VEGFA-165b isoform. It is predominant in

101 many normal tissues, while in melanoma, colorectal and bladder cancer the balance shifts

102 towards highly angiogenic VEGFA-165 variant (Harper \& Bates, 2008; Varey et al., 2008).

103 VEGFA-165b competes with pro-angiogenic isoforms and inhibits VEGFA-165-induced

104 angiogenesis; VEGFA-165b overexpression or administration of recombinant protein inhibits

105 tumor growth in cancer xenograft models indicating its potential as anti-cancer agent (Harper \&

106 Bates, 2008; Varey et al., 2008; Peiris-Pagès, 2012). On the other hand, VEGFA-165b binds to

107 anti-angiogenic drug bevacizumab with equal affinity as VEGFA-165 and thus reduces its

108 efficacy (Varey et al., 2008; Bates et al., 2012).

109 Thus, evaluation of VEGFA isoforms expression pattern in tumor is important for

110 rational choice of anti-angiogenic therapy which is considered to be a promising approach for

111 HCC treatment. However, no quantitative analysis exploring tumor-specific alterations of

112 VEGFA isoforms expression in HCC has been reported yet. The present study aims to compare

113 the full spectrum of VEGFA isoforms expressed in paired HCC and non-tumorous (NT) liver

114 samples and to quantitatively evaluate changes in the expression of major isoforms and their

115 association with tumor clinicopathological characteristics. 
117 Materials \& Methods.

118

119 Clinical samples:

$120 \quad 50 \mathrm{HCC}$ tissue samples and 50 corresponding NT tissue samples were obtained from

121 patients diagnosed with HCC after tumor resection, fresh-frozen in liquid nitrogen and stored at -

$12270^{\circ} \mathrm{C}$. All patients were hepatitis-negative, additional data on clinicopathological parameters are

123 presented in Table 1. HCC diagnosis and origin of samples (tumor or NT tissue) were confirmed

124 by histopathlogical analysis performed by two experienced histopathologists specializing in liver

125 cancer. All tumor samples selected for the study met histological TCGA standards (Nguyen et

126 al., 2011) and contained more than $80 \%$ tumor nuclei and less than $20 \%$ necrotic cells. NT liver

127 samples were taken at least $2 \mathrm{~cm}$ away from tumor margin. All procedures performed were in

128 accordance with Declaration of Helsinki (1964) and its later amendments or comparable ethical

129 standards and were approved by medical ethics committee of FSBI "N.N. Blokhin National

130 Medical Research Center of Oncology" of the Ministry of Health of the Russian Federation.

131 Written informed consent was obtained from all individual participants included in the study.

133 Reverse transcription-polymerase chain reaction (RT-PCR) analysis of VEGFA expression:

134 Reverse transcription was performed using total RNA isolated from $30 \mathrm{mg}$ of tissue with

135 PureLink RNA Mini Kit (Thermo Fisher Scientific, USA) with random hexanucleotides and

136 RevertAid Reverse Transcriptase (Thermo Fisher Scientific, USA).

137 Semi-quantitative RT-PCR analysis of VEGFA isoforms expression spectrum was

138 performed with VEGFA-iso primers flanking variable mRNA region (Fig. 1A). Primer 
139 sequences and RT-PCR conditions are provided in Table S1 and Data S1. TATA-binding protein

140 (TBP) was used as housekeeping gene. PCR products were analyzed by electrophoresis, purified

141 and verified by sequencing (Data S2, Table S2).

142 Quantitative RT-qPCR analysis of VEGFA isoforms expression was performed using

143 primers specifically amplifying different VEGFA isoforms expressed in liver or isoform groups:

144 VEGFA-total (amplifies all VEGFA transcripts), VEGFA-xxxb, VEGFA-189, VEGFA-165,

145 VEGFA-121 (Fig. 1A, Table S1, Table S2). Amount of unspliced VEGFA transcripts containing

146 intron 5 was evaluated using VEGFA-intron5 primers (Table S1, Data S3). PCR conditions were

147 experimentally optimized to achieve reaction efficiency of 98-102\% (Table S1, Data S1).

148 Transcript abundance was estimated using standard samples containing known quantities of

149 corresponding PCR amplicon copies that were obtained by cloning PCR products into pAL2-T

150 vector using Quick-TA kit (Evrogen, Russian Federation) and verified by sequencing (Data S2,

151 Table S2). For each specimen, quantity of VEGFA transcripts was normalized to TBP copy

152 number and changes in relative expression levels and fractions of single isoforms in total pool of

153 VEGFA transcripts were calculated (Data S1).

\section{Statistical analysis:}

156 Differences between observation groups were evaluated using paired sample sign test.

157 Correlations were evaluated using Spearman's rank test, for that numerical clinical parameters

158 (age, tumor size, TNM stage) were used as is, all categorical parameters were assigned rank

159 values (see Table S3 for details). Statistical significance was accepted with $p<0.05$. When

160 analyzing individual cases, 2-fold or stronger changes in gene expression were considered 
161 significant. Statistical analysis and graph plotting were performed using OriginPro 2016 software

162 (OriginLab Corporation, USA).

163

164 Results.

165

166

VEGFA-189, VEGFA-165 and VEGFA-121 are major VEGFA isoforms expressed in liver

tissue:

To explore the full spectrum of VEGFA isoforms expressed in HCC and NT tissue, we performed semi-quantitative RT-PCR analysis using a preliminary panel of $20 \mathrm{HCC}$ cases. We used VEGFA-iso primers that flank the variable region of VEGFA mRNA and amplify several PCR products of different lengths corresponding to certain VEGFA variants (Fig. 1A) The major isoforms expressed in all examined NT and most of HCC specimens were VEGFA-189 (523 bp), VEGFA-165 (451 bp) and VEGFA-121 (319 bp) (Fig. 1, Fig. S1). Isoform identity was verified by sequencing of PCR products (Data S2, Table S2). Decrease in VEGFA-189 level was the predominant aberration of VEGFA isoforms expression found in HCC samples; we also observed occasional up- or downregulation of VEGFA-165 and VEGFA-121 (Fig. 1B). No prominent bands corresponding to other VEGFA variants were detected.

\section{VEGFA-189 isoform expression is frequently downregulated in HCC:}

To further explore changes in VEGFA variants expression, we performed quantitative

181 RT-qPCR-analysis of expanded set comprising $50 \mathrm{HCC}$ cases with primers specific to VEGFA

182 isoforms or isoform groups (Table S1, Table S2). Raw data obtained in RT-qPCR analysis for

183 each examined sample are presented in Data S4. VEGFA-total primers were used to evaluate the 
184 amount of all VEGFA isoforms expressed in examined samples (Fig. 1A); however, certain

185 fraction of all VEGFA transcripts retains unspliced intron 5 sequence that can be detected using

186 VEGFA-intron5 primers (Table S2, Data S3). The fraction of unspliced transcripts in examined

187 samples varied from $0.1 \%$ to $34.7 \%$ (Fig. S2). We therefore calculated the amount of all mature

188 VEGFA transcripts (referred to as "VEGFA-spliced") by subtracting VEGFA-intron5 quantity

189 from that of VEGFA-total (Data S3). Analysis of single cases revealed considerable

190 heterogeneity of VEGFA-spliced changes (both in direction and magnitude), no significant

191 changes in VEGFA-spliced expression were detected between HCC and NT sample sets. Only

19211 cases of $50(22 \%)$ displayed more than 2-fold change of VEGFA-spliced expression (Fig. 193 2A).

194 Using primers specific to anti-angiogenic VEGFA-xxxb isoforms we demonstrated that 195 their fraction in examined samples does not exceed 5\% of all spliced VEGFA transcripts. While 196 higher than 2-fold changes in VEGFA-xxxb expression level are present in part of examined 197 cases (Fig. S3), there is no significant difference between VEGFA-xxxb expression levels in NT 198 and HCC sample sets (Fig. 2B). Due to these facts we focused further studies on expression of 199 pro-angiogenic isoforms VEGFA-189, VEGFA-165 and VEGFA-121 using isoform-specific 200 primers.

201 To verify that our approach to VEGFA isoform quantitation is correct, we compared the 202 sum of copy numbers obtained for VEGFA-189, VEGFA-165 and VEGFA-121 isoforms with 203 VEGFA-spliced copy number in every examined sample. The average ratio of these values 204 across the panel was $99.8 \%$ (95\% confidence interval: 97.1\% - 102.5\%) indicating that VEGFA205 189, VEGFA-165 and VEGFA-121 are indeed the main components of total VEGFA transcripts 206 pool in examined specimens. 
sample set, while no significant changes were observed in VEGFA-165 and VEGFA-121

210 in HCC ( $\geq 2$-fold downregulation was detected in $56 \%$ cases) while VEGFA-165 and VEGFA-

211121 variants displayed significant changes in smaller fraction of cases $(22 \%$ and $32 \%$,

212 respectively) (Fig. 2C-E). Hierarchical clustering revealed that decrease in total VEGFA-spliced 213 expression occurs mostly due to VEGFA-189 repression while its increase is associated with 214 VEGFA-165 and VEGFA-121 upregulation (Fig. 2F).

\section{Shift of VEGFA isoforms balance occurs in HCC tissue and is associated with tumor}

\section{7 clinicopathological characteristics:}

Since different VEGFA-xxxa isoforms exhibit different angiogenesis-stimulating

219 properties, shift of their balance can considerably modulate VEGFA signalization. Thus the cumulative impact of several VEGFA isoforms depends not only on their absolute expression levels, but also on proportions between particular variants. We therefore decided to estimate the ratios between VEGFA-189, VEGFA-165 and VEGFA-121. Since the amount of other VEGFA variants in examined samples is negligible in comparison to the amount of three predominant

224 isoforms, we used simplified calculation model considering the amount of all mature VEGFA 225 transcripts as a sum of VEGFA-189, VEGFA-165 and VEGFA-121 copy numbers in each

226 individual sample and calculated fractions of each of the predominant isoforms (See Data S4).

227 Significant decrease in VEGFA-189 fraction and corresponding increase in fractions of VEGFA228165 and VEGFA-121 were observed in HCC tissue (Fig. 3A-D). Cluster analysis identified two 
229 distinct subsets of cases in which reduction in VEGFA-189 fraction is complemented by increase

230 in either VEGFA-165 or VEGFA-121 fraction (Fig. 3E).

231 Correlation analysis revealed that changes in VEGFA isoform fractions were stronger

232 associated with clinically significant tumor characteristics than alterations in expression levels

233 (Table 2). Increase in expression levels of VEGFA-spliced, VEGFA-165 or VEGFA-121

234 correlated with ascites presence and feeble or absent tumor capsule. In contrast, reduction of

235 VEGFA-189 fraction and increase in VEGFA-121 fraction were associated with advanced tumor

236 stages estimated using Tumor-Node-Metastasis (TNM) (Edge et al., 2010) or Barcelona Clinic

237 Liver Cancer (BCLC) (Bruix, Reig \& Sherman, 2016) systems. Decrease in VEGFA-189

238 fraction was also associated with poor HCC differentiation and higher level of serum AFP.

240 Discussion.

There is growing evidence that aberrations in alternative splicing play significant role in carcinogenesis. Splice isoforms may possess different functional properties defining their pro- or anti-oncogenic activity (Ladomery, 2013; Berasain et al., 2014; Elizalde et al., 2014). Spectrum and ratios of expressed VEGFA isoforms are tissue-specific and provide the establishment of vascular network matching specific tissue functions ( $\mathrm{Ng}$ et al., 2001; Vempati,

247 Popel \& Mac Gabhann, 2014). Difference in physiological availability and receptor interactions 248 of alternative VEGFA variants modulates their angiogenesis-stimulating potential (Plouët et al.,

249 1997; Ferrara, Gerber \& LeCouter, 2003; Rapisarda \& Melillo, 2012; Vempati, Popel \& Mac

250 Gabhann, 2014). VEGFA is an important regulator of HCC progression and was previously

251 reported to be overexpressed in HCC tissue, so aberrations in isoform expression balance may 
252 directly affect tumor development (Tseng et al., 2008; Shen, Hsu \& Cheng, 2010; Chekhonin et 253 al., 2013). However, most studies regarding the role of VEGFA in HCC development estimate

254 only total expression level, while the data on VEGFA isoforms expression in liver tumors is still

255 incomplete and contradictory. In present study we used paired HCC and NT tissue samples to

256 quantitatively evaluate tumor-specific changes in expression levels of VEGFA isoforms, ratios

257 between them and correlations of these changes with clinically relevant tumor properties.

258 While VEGFA is known to be frequently overexpressed in various tumors (Ferrara,

259 Gerber \& LeCouter, 2003; Chekhonin et al., 2013), our results indicate considerable

260 heterogeneity of total VEGFA expression alterations in HCC. Similar results based on

261 immunohistochemical staining were reported (Tseng et al., 2008) and explain lack of significant

262 difference in VEGFA-spliced expression between whole NT and HCC sample sets.

263 Anti-angiogenic VEGFA-xxxb variants, which are predominant in several normal tissues

264 (Harper \& Bates, 2008; Varey et al., 2008), comprise a very small fraction of all VEGFA

265 transcripts in examined samples (Fig. 2B). Anti-angiogenic action of VEGFA-xxxb variants is

266 supposed to be carried out by competing with VEGFA-xxxa for binding to VEGF receptors.

267 Since VEGFA-xxxb fraction is negligibly small not only in HCC, but also in NT tissue, we

268 suppose that VEGFA-xxxb isoforms do not exert significant impact upon VEGFA signaling in

269 the liver.

270 In agreement with previously published data, VEGFA-189, VEGFA-165 and VEGFA-

271121 isoforms were the major variants expressed in non-cancerous liver ( $\mathrm{Ng}$ et al., 2001; Sheen et

272 al., 2005; Li et al., 2006; Iavarone et al., 2007). All three variants were reported to be

273 overexpressed in HCC tissue compared to independent (VEGFA-189, VEGFA-165, VEGFA-

274 121) or paired (VEGFA-165) NT specimens (Jeng et al., 2004; Li et al., 2006; Iavarone et al., 
275 2007). Surprisingly, most HCC samples examined in present study displayed decrease in

276 VEGFA-189 expression, while VEGFA-165 and VEGFA-121 variants could be either up- or

277 downregulated (Fig. 2C-E). We attribute this inconsistence with previous studies to different

278 etiology of HCC samples used in present study (paired samples, hepatitis-negative cases only),

279 the usage of isoform-specific primers instead of universal ones and limitations of semi-

280 quantitative conventional RT-PCR approach used in previous studies. This is the first time

281 VEGFA-189 is demonstrated to be repressed in the majority of HCC cases indicating that its role

282 in HCC progression may be significantly different from that of VEGFA-165 and VEGFA-121.

283 As stated above, the activity of VEGFA-induced signaling can be affected not only by

284 changes in individual isoforms expression, but also by changes in their balance. The shift of

285 isoforms ratio from poorly diffusible VEGFA-189 variants towards more angiogenic VEGFA-

286165 and VEGFA-121 ones observed in HCC tissue is much more prominent than alterations of

287 expression levels of corresponding isoforms. There are two possible causes of VEGFA-189

288 fraction reduction: direct VEGFA-189 downregulation or increase in VEGFA-165 and VEGFA-

289121 expression levels. According to our results, these two alterations rarely occur simultaneously

290 (Fig. 2F) thus implying that mechanisms controlling VEGFA-189 expression may be

291 considerably different from ones regulating expression of VEGFA-165 and VEGFA-121. This

292 hypothesis is further supported by the fact that reduction in VEGFA-189 fraction is accompanied

293 by increase in fraction of either VEGFA-165 or VEGFA-121 but rarely both (Fig. 3E). Our data

294 indicate that splicing of VEGFA pre-mRNA is controlled by a very elaborate mechanism.

295 Supposedly, HCC development is accompanied by deregulation of this mechanism that results in

296 VEGFA pre-mRNA being preferably processed into VEGFA-165 and VEGFA-121 variants 
297 instead of VEGFA-189, but available information on VEGFA splicing mechanisms is

298 insufficient for making more explicit statement.

299 The importance of VEGFA isoforms fractions evaluation is clearly illustrated by the fact

300 that changes in VEGFA isoform fractions, but not in their expression levels, are associated with

301 such essential HCC clinicopathological features as TNM and BCLC stages, the latter currently

302 being considered the most effective system for HCC prognosis and treatment optimization (Edge

303 et al., 2010; Bruix, Reig \& Sherman, 2016). Moreover, prominent reduction of VEGFA-189

304 fraction is associated with more aggressive tumor phenotype (advanced TNM and BCLC stages,

305 poor differentiation level and higher serum AFP level) further supporting our hypothesis of

306 distinct role of VEGFA-189 downregulation in HCC progression and implying possible tumor-

307 suppressive functions of VEGFA-189.

308 Increase in VEGFA-165 and VEGFA-121 expression levels is associated with ascites and

309 feeble tumor capsule indicating their progression-stimulating role consistent with previously

310 reported data (Sheen et al., 2005). High levels of pro-angiogenic VEGFA isoforms expression

311 may contribute to sensitivity of tumor to anti-VEGFA drugs like bevacizumab (Bates et al.,

312 2012). However, VEGFA-121 overexpression can reduce tumor sensitivity to inhibitors of KDR

313 receptor, particularly sorafenib, since VEGFA-121 mainly acts through alternative FLT-1

314 receptor (Rapisarda \& Melillo, 2012; Vempati, Popel \& Mac Gabhann, 2014).

315 Data on VEGFA-189 functions in different tumors are rather controversial. This isoform

316 was reported to be overexpressed in colon, ovary and lung tumors, while its repression was

317 described in non-small cell lung carcinoma (Vempati, Popel \& Mac Gabhann, 2014). On the one

318 hand, VEGFA-189, like other VEGFA-xxxa isoforms, may exert pro-oncologic effects. Its

319 overexpression can promote migration of breast cancer cells and is associated with colon cancer 
320 and lung cancer metastases (Tokunaga et al., 1998; Nishi et al., 2005; Hervé et al., 2008).

321 VEGFA-189 stimulates the growth of colon tumors in vivo, but to a lesser extent than VEGFA-

322165 (Tomii et al., 2002). On the other hand, in breast cancer VEGFA-189 may possess anti-

323 tumor functions since it reduces invasion and metastatic potential of tumor cells and promotes

324 apoptosis in stress conditions (Vintonenko et al., 2011; Di Benedetto et al., 2015). It also exerts

325 opposite effects on proliferation of endothelial cells originating from different tissues (Hervé et

326 al., 2005). Unlike VEGFA-164 and VEGFA-120, VEGFA-188 (mouse counterparts of human

327 VEGFA-165, VEGFA-121 and VEGFA-189 isoforms) does not affect fibrosarcoma cells

328 proliferation and migration, but induces apoptosis (Kanthou et al., 2014). It is likely that

329 VEGFA-189 functions are tissue-specific and can differ considerably from those of VEGFA-165

330 and VEGFA-121 since unprocessed VEGFA-189 is much weaker stimulator of angiogenesis

331 and, possibly, acts via distinct signaling pathways (Plouët et al., 1997; Vempati, Popel \& Mac

332 Gabhann, 2014). We suppose that reduction of VEGFA-189 fraction in HCC tissue can

333 contribute to the development of more aggressive tumor phenotype. Possibly such tumors could

334 be more sensitive to anti-VEGFA treatment like bevacizumab therapy due to increased fractions

335 of VEGFA-165 and VEGFA-121.

336 Current studies of the role of VEGFA splice variants in cancer development are mainly

337 focused on balance of pro- and anti-angiogenic VEGFA isoform groups. Up to date several

338 regulators of VEGFA mRNA splicing are identified (SRp40, SRp55, SRSF1) that interact with

339 exon 8 thus controlling generation of VEGFA-xxxa or VEGFA-xxxb variants (Nowak et al.,

340 2008; Nowak et al., 2010). However, limited information is available on possible regulators of

341 VEGFA mRNA splicing events involving exons 5-7 that result in generation of VEGFA-189,

342 VEGFA-165 and VEGFA-121. SRp20, SRp40 and SRSF1 proteins were reported to be 
343 connected to hypoxia-induced shift in VEGFA isoform balance towards VEGFA-121, but their

344 ability to directly interact with sequence of exons 5-7 is yet to be investigated (Elias \& Dias,

345 2008) . VEGFA-xxxa splicing can also be influenced by non-coding RNAs (MALAT1) and

346 chromatin modifiers (EHMT2); the latter can specifically prevent the inclusion of exon 6a into

347 VEGFA mRNA thus shifting the balance from VEGFA-189 towards VEGFA-165 (Salton, Voss

348 \& Misteli, 2014; Pruszko et al., 2017).

349 Since there is no reliable experimental approach to induce VEGFA splicing changes,

350 most of published reports on functions of VEGFA-xxxa isoforms evaluate effects caused by

351 overexpression of certain VEGFA variants (Tomii et al., 2002; Hervé et al., 2008; Vintonenko et

352 al., 2011; Kanthou et al., 2014; Di Benedetto et al., 2015). Whereas inactivation of a single

353 VEGFA isoform is much more complicated task, there is only one published investigation

354 describing ribozyme-mediated specific cleavage of VEGFA-189 in non-small cell lung cancer

355 cells that resulted in attenuation of their malignant potential (Oshika et al., 2000). Given possible

356 tissue specificity of VEGFA-189 functions, additional experiments on its inactivation are

357 necessary to determine possible clinical impact of VEGFA-189 and its role in tumor

358 development and angiogenesis. Implementation of highly specific RNA interference approaches

359 to selectively knockdown VEGFA-189 expression and identification of VEGFA exon 6 splicing

360 regulators could provide valuable information essential for achieving this goal.

361

362 Conclusions.

363

364 Using quantitative approach, we have detected HCC-specific shift of VEGFA isoforms

365 ratios that consisted in decrease in VEGFA-189 and increase in VEGFA-165 and VEGFA-121 
366 fractions. These changes were associated with multiple essential clinicopathological tumor

367 characteristics. The clinical significance of presented data consists in their potent impact on 368 optimization of HCC treatment since VEGFA isoforms ratio may be a promising factor for

369 prediction of anti-angiogenic therapy efficiency. Further studies of VEGFA isoforms expression,

370 VEGFA mRNA splicing regulation and VEGFA-189 functional properties in HCC are necessary

371 to evaluate its possible association with other tumor progression factors, survival and recurrence.

372

373 Acknowledgements.

374

375

376

377 experiments.

378

379

\section{References.}

382 Meropol NJ, Benson AB, Harper SJ. 2012. Association between VEGF splice isoforms and 383 progression-free survival in metastatic colorectal cancer patients treated with bevacizumab.

384 Clinical Cancer Research 18:6384-6391. DOI: 10.1158/1078-0432.CCR-12-2223.

Prieto J, Avila MA. 2014. Alterations in the expression and activity of pre-mRNA splicing

387 factors in hepatocarcinogenesis. Hepatic Oncology 1:241-252. DOI: 10.2217/hep.13.17. 
Bruix J, Qin S, Merle P, Granito A, Huang YH, Bodoky G, Pracht M, Yokosuka O,

389 Rosmorduc O, Breder V, Gerolami R, Masi G, Ross PJ, Song T, Bronowicki JP, Ollivier-

390 Hourmand I, Kudo M, Cheng AL, Llovet JM, Finn RS, LeBerre MA, Baumhauer A, Meinhardt

391 G, Han G; RESORCE Investigators. 2017. Regorafenib for patients with hepatocellular

392 carcinoma who progressed on sorafenib treatment (RESORCE): a randomised, double-blind,

393 placebo-controlled, phase 3 trial. Lancet 389:56-66. DOI: 10.1016/S0140-6736(16)32453-9.

394 Bruix J, Reig M, Sherman M. 2016. Evidence-Based Diagnosis, Staging, and Treatment

395 of Patients With Hepatocellular Carcinoma. Gastroenterology 150:835-853. DOI:

$39610.1053 /$ j.gastro.2015.12.041.

397 Chekhonin VP, Shein SA, Korchagina AA, Gurina OI. 2013. VEGF in tumor progression

398 and targeted therapy. Current Cancer Drug Targets 13:423-443. DOI:

399

$10.2174 / 15680096113139990074$.

400 Di Benedetto M, Toullec A, Buteau-Lozano H, Abdelkarim M, Vacher S, Velasco G,

401 Christofari M, Pocard M, Bieche I, Perrot-Applanat M. 2015. MDA-MB-231 breast cancer cells

402 overexpressing single VEGF isoforms display distinct colonisation characteristics. British

403 Journal of Cancer 113:773-785. DOI: 10.1038/bjc.2015.267.

404 Edge SB, Byrd DR, Compton CC, Fritz AG, Greene FL, Trotti, III, A. 2010. AJCC

405 Cancer Staging Manual, 7th Ed. New York: Springer.

406 Elias AP, Dias S. 2008. Microenvironment changes (in pH) affect VEGF alternative

407 splicing. Cancer Microenvironment 1:131-139. DOI: 10.1007/s12307-008-0013-4.

408 Elizalde M, Urtasun R, Azkona M, Latasa MU, Goñi S, García-Irigoyen O, Uriarte I,

409 Segura V, Collantes M, Di Scala M, Lujambio A, Prieto J, Ávila MA, Berasain C. 2014. Splicing 
410 regulator SLU7 is essential for maintaining liver homeostasis. The Journal of Clinical

411 Investigations 124:2909-2920. DOI: 10.1172/JCI74382.

412 Ferrara N, Gerber HP, LeCouter J. 2003. The biology of VEGF and its receptors. Nature 413 Medicine 9:669-676. DOI: 10.1038/nm0603-669.

414 Harper SJ, Bates DO. 2008. VEGF-A splicing: the key to anti-angiogenic therapeutics?

415 Nature Reviews Cancer 8:880-887. DOI: doi: 10.1038/nrc2505.

416 Hervé MA, Buteau-Lozano H, Mourah S, Calvo F, Perrot-Applanat M. 2005. VEGF189

417 stimulates endothelial cells proliferation and migration in vitro and up-regulates the expression

418 of Flk-1/KDR mRNA. Experimental Cell Research, 309:24-31. DOI:

419

10.1016/j.yexcr.2005.05.022.

420 Hervé MA, Buteau-Lozano H, Vassy R, Bieche I, Velasco G, Pla M, Perret G, Mourah S, 421 Perrot-Applanat M. 2008. Overexpression of vascular endothelial growth factor 189 in breast 422 cancer cells leads to delayed tumor uptake with dilated intratumoral vessels. The American 423 Journal of Pathology 172:167-178. DOI: 10.2353/ajpath.2008.070181.

424 Iavarone M, Lampertico P, Iannuzzi F, Manenti E, Donato MF, Arosio E, Bertolini F, 425 Primignani M, Sangiovanni A, Colombo M. 2007. Increased expression of vascular endothelial 426 growth factor in small hepatocellular carcinoma. Journal of Viral Hepatitis 14:133-139. DOI: $427 \quad 10.1111 / j .1365-2893.2006 .00782 . x$.

428 Jeng KS, Sheen IS, Wang YC, Gu SL, Chu CM, Shih SC, Wang PC, Chang WH, Wang 429 HY. 2004. Is the vascular endothelial growth factor messenger RNA expression in resectable 430 hepatocellular carcinoma of prognostic value after resection? World Journal of Gastroenterology 431 10:676-681. DOI: 10.3748/wjg.v10.i5.676. 
433 Santos SA, Reyes-Aldasoro CC, English WR, Tozer GM. 2014. Tumour cells expressing single

434 VEGF isoforms display distinct growth, survival and migration characteristics. PLoS One

435 9:e104015. DOI: 10.1371/journal.pone.0104015.

436 Ladomery M. 2013. Aberrant alternative splicing is another hallmark of cancer,

437 International Journal of Cell Biology 2013:463786. DOI: 10.1155/2013/463786.

438 Li Q, Xu B, Fu L, Hao XS. 2006. Correlation of four vascular specific growth factors

439 with carcinogenesis and portal vein tumor thrombus formation in human hepatocellular

440 carcinoma, Journal of Experimental \& Clinical Cancer Research 25:403-409.

Liu K, Zhang X, Xu W, Chen J, Yu J, Gamble JR, McCaughan GW. 2017. Targeting the

vasculature in hepatocellular carcinoma treatment: Starving versus normalizing blood supply.

Clinical and Translational Gastroenterology 8:e98. DOI: 10.1038/ctg.2017.28.

Llovet JM, Zucman-Rossi J, Pikarsky E, Sangro B, Schwartz M, Sherman M, Gores G.

2016. Hepatocellular carcinoma. Nature Reviews Disease Primers 2:16018. DOI:

10.1038/nrdp.2016.18.

Ng YS, Rohan R, Sunday ME, Demello DE, D’Amore PA. 2001. Differential expression

of VEGF isoforms in mouse during development and in the adult. Developmental Dynamics

220:112-121. DOI: 10.1002/1097-0177(2000)9999:9999<::AID-DVDY1093>3.0.CO;2-D.

Nguyen NT, Cotton RT, Harring TR, Guiteau JJ, Gingras MC, Wheeler DA, O'Mahony

451 CA, Gibbs RA, Brunicardi FC, Goss JA. 2011. A primer on a hepatocellular carcinoma

452 bioresource bank using the cancer genome atlas guidelines: practical issues and pitfalls. World 453 Journal of Surgery 35:1732-1737. DOI: 10.1007/s00268-010-0953-y. 
Inoue H, Ueyama Y, Nakamura M. 2005. Cell binding isoforms of vascular endothelial growth

International Journal of Oncology 26:1517-1524. DOI: 10.3892/ijo.26.6.1517. endothelial growth factor (VEGF) splicing from pro-angiogenic to anti-angiogenic isoforms: a novel therapeutic strategy for angiogenesis. Journal of Biological Chemistry 285:5532-5540.

DOI: $10.1074 /$ jbc.M109.074930. 20):3487-3495. DOI: $10.1242 /$ jcs.016410. vascular endothelial growth factor (VEGF) 189 expression in non-small cell lung cancer (NSCLC). European Journal of Cancer 36:2390-2396. DOI: 10.1016/S0959-8049(00)00343-9. Peiris-Pagès M. 2012. The role of VEGF 165b in pathophysiology, Cell Adhesion \& Migration 6:561-568. DOI: 10.4161/cam.22439. 13396. 
478 F, Bates DO, Bicciato S, Zylicz M, Zylicz A, Blandino G, Fontemaggi G. 2017. The mutant p53-

479 ID4 complex controls VEGFA isoforms by recruiting lncRNA MALAT1. EMBO Reports

480 18:1331-1351. DOI: 10.15252/embr.201643370.

481 Rapisarda A, Melillo G. 2012. Role of the VEGF/VEGFR axis in cancer biology and 482 therapy. Advances in Cancer Research 114:237-267. DOI: 10.1016/B978-0-12-386503-8.000064835.

Salton M, Voss TC, Misteli T. 2014. Identification by high-throughput imaging of the 485 histone methyltransferase EHMT2 as an epigenetic regulator of VEGFA alternative splicing. 486 Nucleic Acids Research 42:13662-13673. DOI: 10.1093/nar/gku1226. endothelial growth factor mRNA in noncancerous liver remnants of patients with hepatocellular carcinoma. World Journal of Gastroenterology 11:187-192. DOI: 10.3748/wjg.v11.i2.187. 45:794-807. DOI: 10.1007/s00535-010-0270-0. mRNA isoform expression pattern is correlated with liver metastasis and poor prognosis in colon cancer. British Journal of Cancer 77:998-1002. DOI: 10.1038/bjc.1998.164. 
500189 amino acid isoform of vascular endothelial growth factor in tumorigenesis. International

501 Journal of Oncology 21:1251-1257. DOI: 10.3892/ijo.21.6.1251.

502 Tseng PL, Tai MH, Huang CC, Wang CC, Lin JW, Hung CH, Chen CH, Wang JH, Lu

503 SN, Lee CM, Changchien CS, Hu TH. 2008. Overexpression of VEGF is associated with

504 positive p53 immunostaining in hepatocellular carcinoma (HCC) and adverse outcome of HCC

505 patients. Journal of Surgical Oncology 98:349-357. DOI: 10.1002/jso.21109.

506

Varey AH, Rennel ES, Qiu Y, Bevan HS, Perrin RM, Raffy S, Dixon AR, Paraskeva C,

507 Zaccheo O, Hassan AB, Harper SJ, Bates DO. 2008. VEGF 165 b, an antiangiogenic VEGF-A

508 isoform, binds and inhibits bevacizumab treatment in experimental colorectal carcinoma: balance

509 of pro- and antiangiogenic VEGF-A isoforms has implications for therapy. British Journal of

510 Cancer 98:1366-1379. DOI: 10.1038/sj.bjc.6604308.

511 Vempati P, Popel AS, Mac Gabhann F. 2014. Extracellular regulation of VEGF:

512 isoforms, proteolysis, and vascular patterning, Cytokine \& Growth Factor Reviews 25:1-19.

513 DOI: 10.1016/j.cytogfr.2013.11.002.

514 Vintonenko N, Pelaez-Garavito I, Buteau-Lozano H, Toullec A, Lidereau R, Perret GY,

515 Bieche I, Perrot-Applanat M. 2011. Overexpression of VEGF189 in breast cancer cells induces

516 apoptosis via NRP1 under stress conditions, Cell Adhesion \& Migration 5:332-343. DOI:

517 10.4161/cam.5.4.17287.

518 Wörns MA, Galle PR. 2010. Future perspectives in hepatocellular carcinoma, Digestive

519 and Liver Disease 42 Suppl 3:S302-309. DOI: 10.1016/S1590-8658(10)60521-X. 


\section{Figure 1}

Spectrum of VEGFA isoforms expressed in HCC and NT liver tissue.

(A) VEGFA isoforms generated through alternative splicing affecting exons 6, 7 and 8.

Numbers 1-8 designate exons, arrows indicate primer annealing sites. PCR product lengths are indicated for VEGFA-iso primers, bp - base pairs. (B) Representative results reflecting VEGFA isoforms expression spectrum determined by RT-PCR analysis. M - 100bp DNA marker, NT - non-tumor sample, T - tumor sample. Case numbers are indicated above.

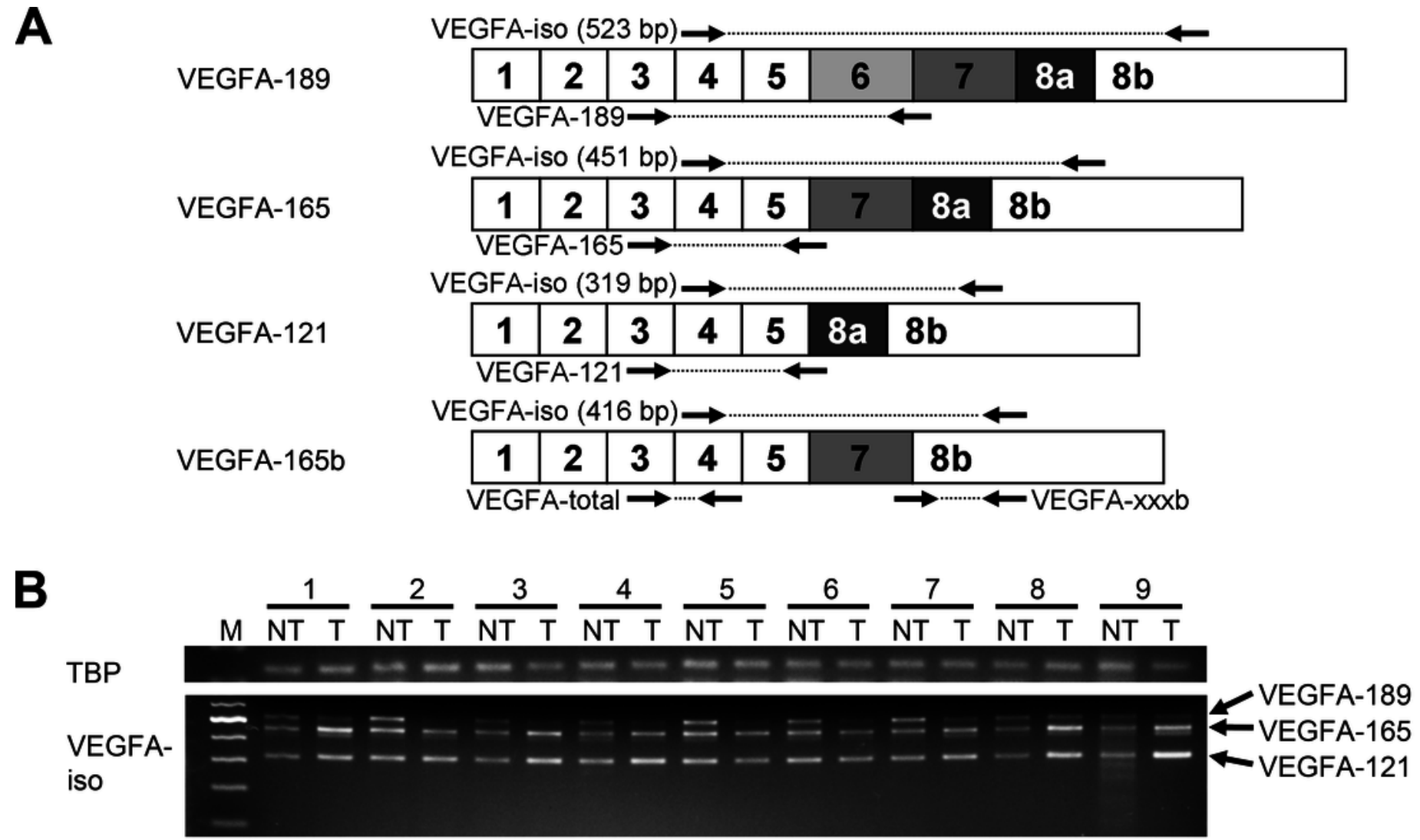




\section{Figure 2}

Alterations of expression levels of VEGFA isoforms in HCC tissue samples in comparison to corresponding NT samples.

(A) Changes in expression level of all mature VEGFA transcripts. (B) VEGFA-xxxb fractions in all examined samples and expression levels in NT and HCC sample sets. (C-E) Changes in expression levels of VEGFA-189 (C), VEGFA-165 (D) and VEGFA-121 (E). (F) Cluster analysis of changes in VEGFA isoforms expression levels. Data for NT or HCC sample sets $(n=50)$ or all samples set $(n=100)$ are presented as box-and-whisker plots, data for individual cases are presented as NT/HCC ratios in logarithmic scale. NS - non-significant difference. 

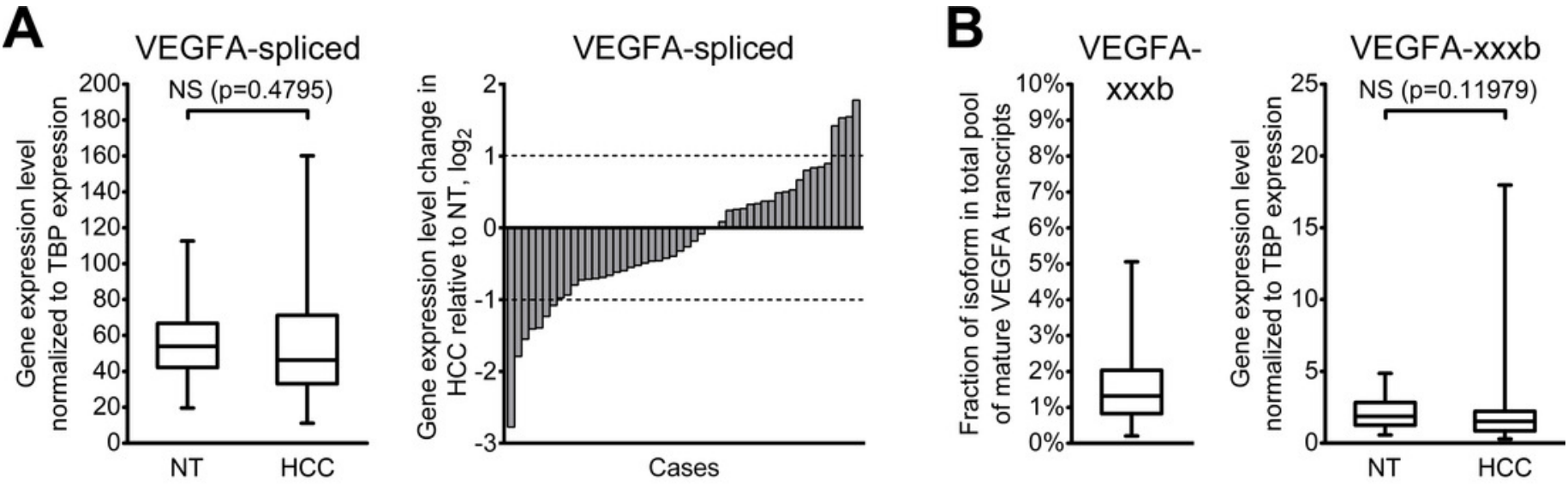

C

VEGFA-189

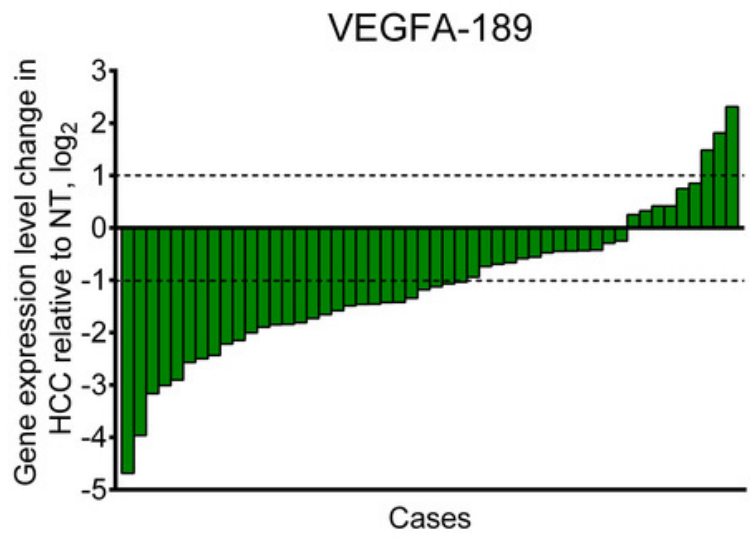

$\mathbf{F}$

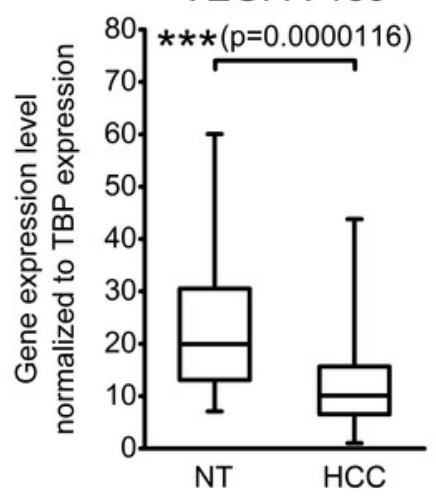

D

VEGFA-165

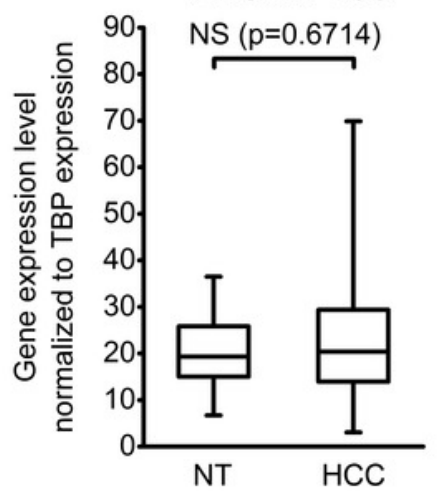

$\mathbf{E}$
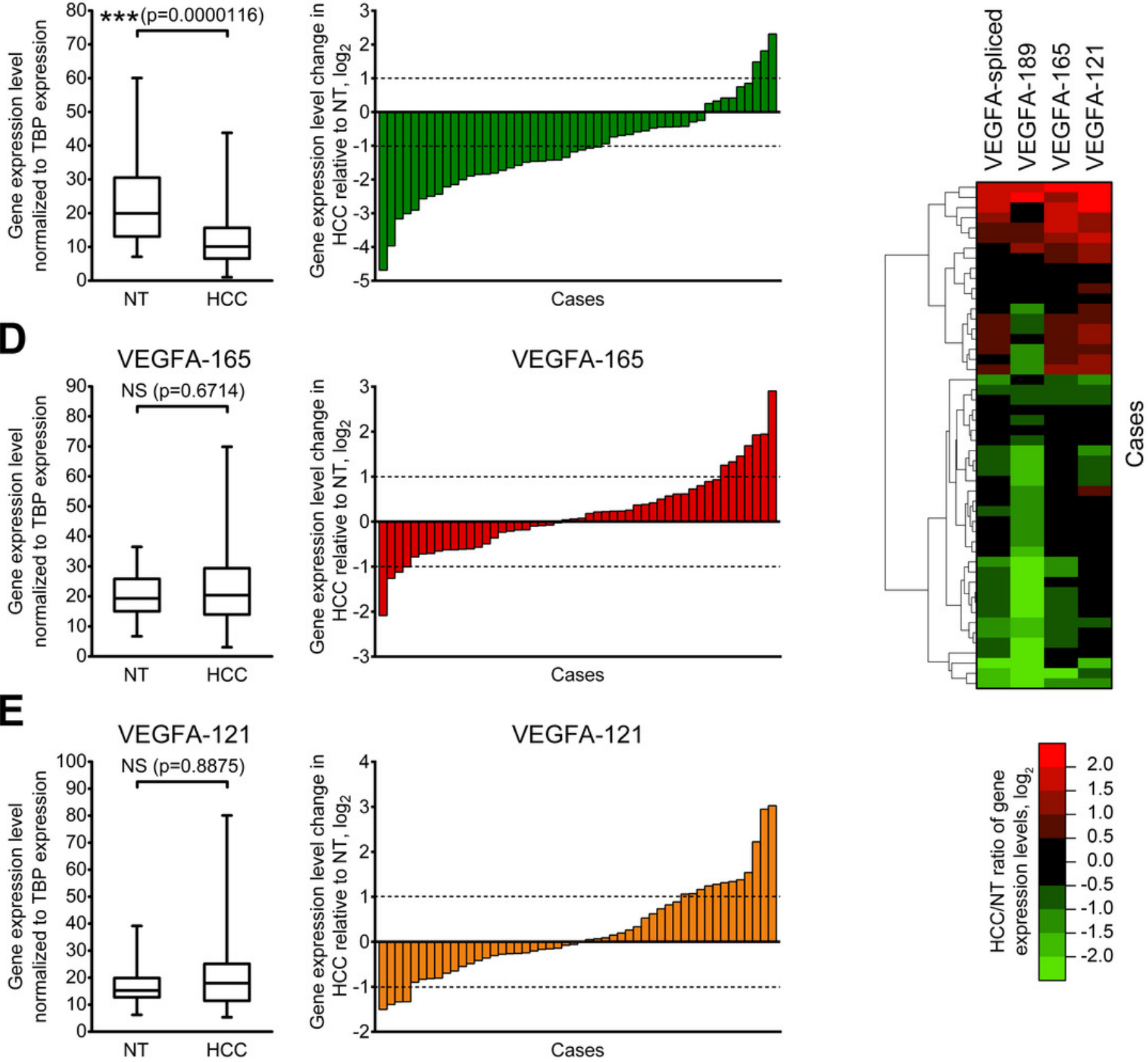


\section{Figure 3}

Alterations of fractions of VEGFA-189, VEGFA-165 and VEGFA-121 in total pool of mature VEGFA transcripts.

(A) Average proportions of major VEGFA variants in HCC and NT tissue. (B-D) Changes in fractions of VEGFA-189 (B), VEGFA-165 (C) and VEGFA-121 (D) in total pool of mature VEGFA transcripts. Order of cases presented on dot-plots is rearranged for each isoform to better reflect change trends. (E) Cluster analysis of changes in VEGFA fractions. Data for whole NT and HCC sample sets $(n=50)$ are presented as box-and-whiskers plots of isoform fractions, data for individual cases are presented as isoform fractions (dot-plots) or differences between paired HCC and NT samples in linear scale (heatmap plot). NS - non-significant difference. 
A
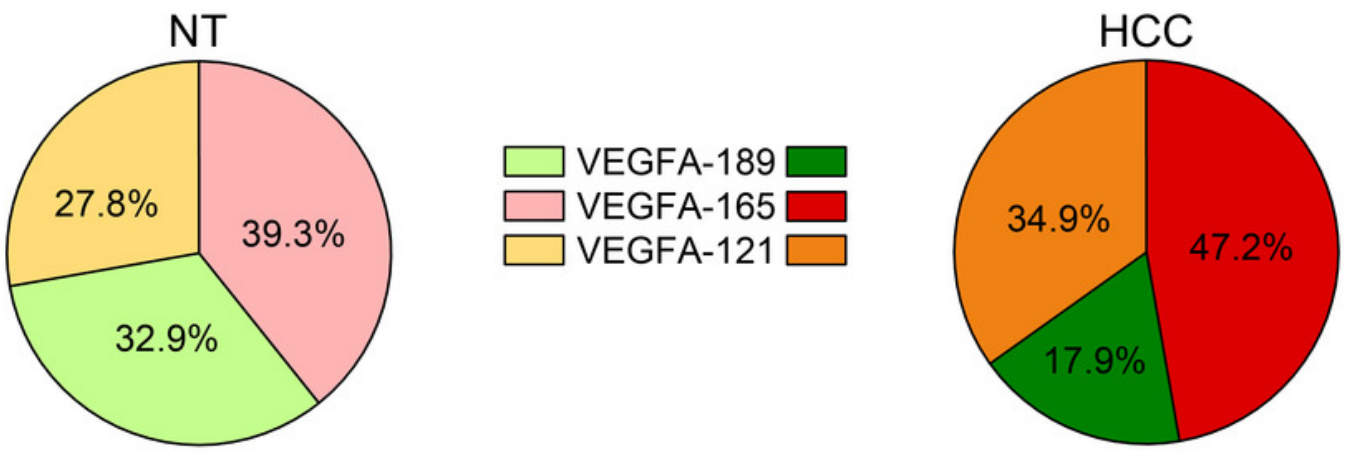

B

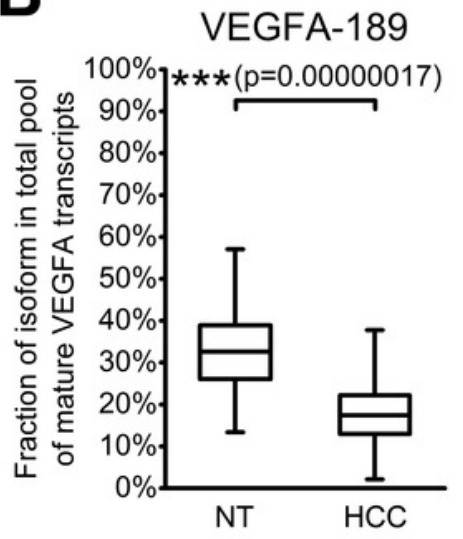

c

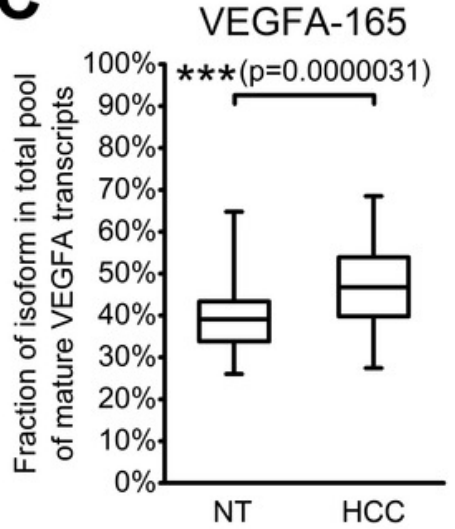

D

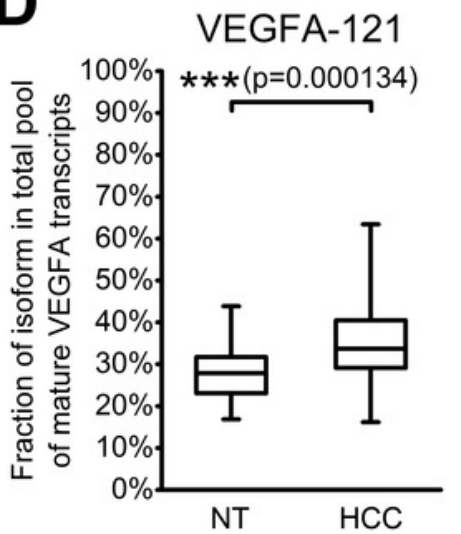

VEGFA-189

VEGFA-165

VEGFA-121

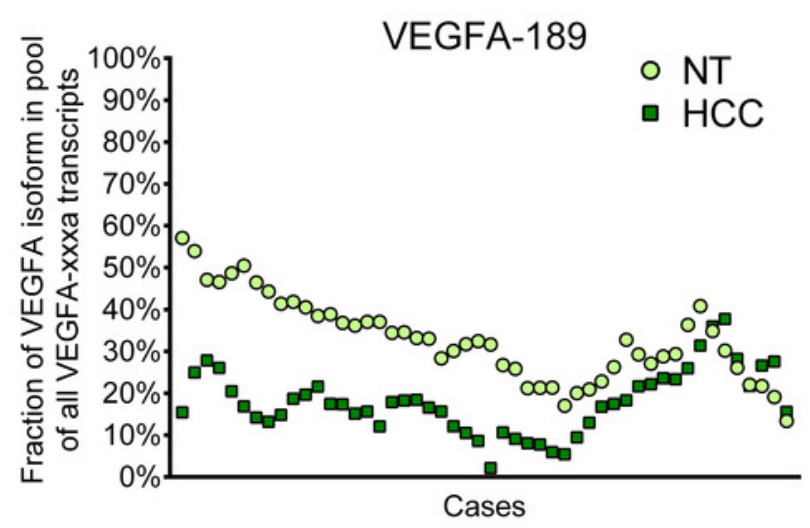

VEGFA-165

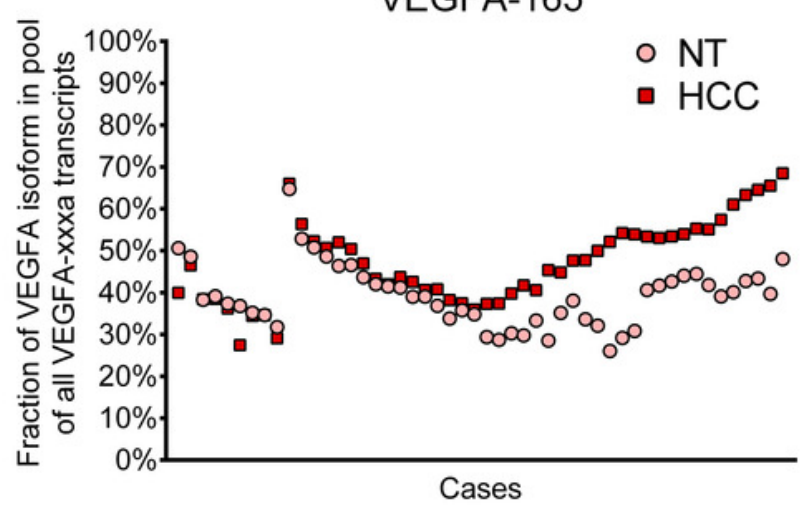

VEGFA-121

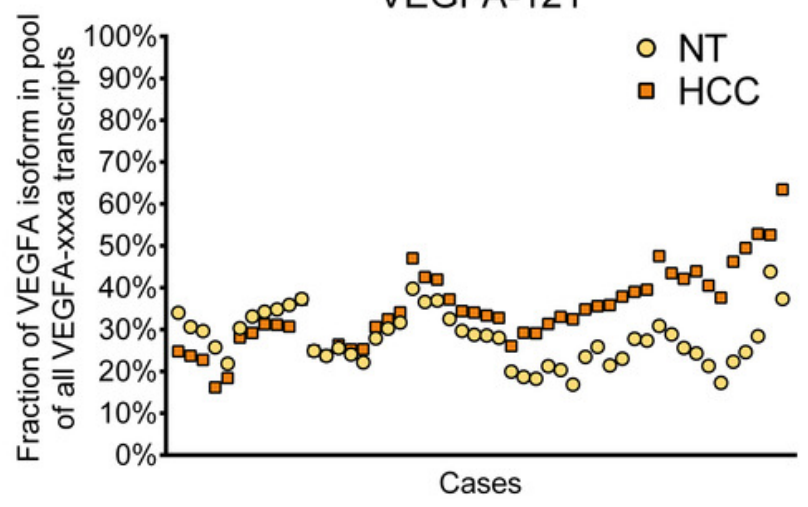

$\mathbf{E}$

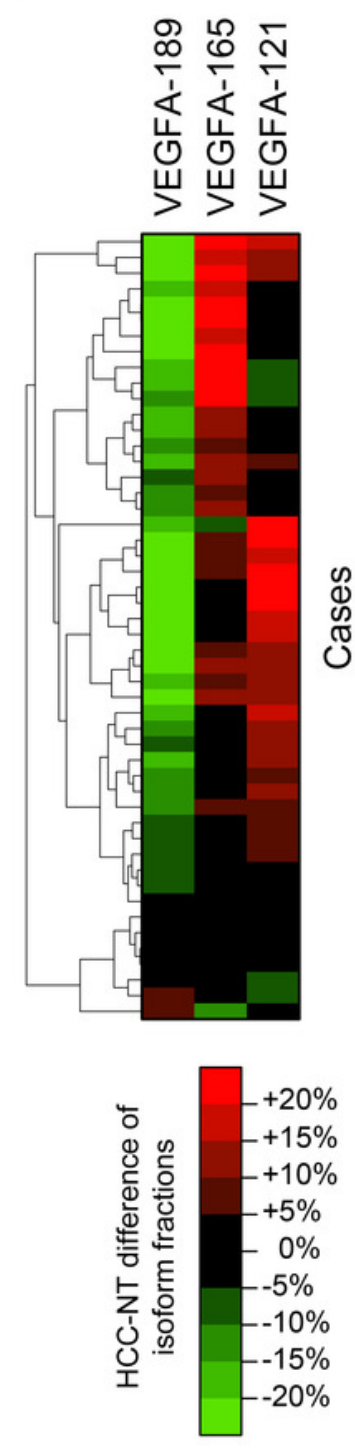




\section{Table 1 (on next page)}

Clinicopathological characteristics of examined HCC cases. 
Characteristic

Age, years (mean \pm SD)

Gender, male / female

TNM staging, I / II / III / IV

BCLC staging, A / B / C / D

Tumor size, $\mathrm{cm}($ mean $\pm \mathrm{SD})$

Intrahepatic metastases, yes / no

Lymph node metastases, yes / no

Distant metastases, yes / no

Tumor capsule presence, absent / feeble / prominent / N/A ${ }^{\mathrm{a}}$

Invasion into blood vessels, yes / no

Tumor vascularity ${ }^{\mathrm{b}}$, low / moderate / high / N/A

Histological differentiation, Edmondson-Steiner grade, G1 / G2 / G3 / G4 / Gx ${ }^{\mathrm{c}}$

Alpha-fetoprotein serum level, low $(<50 \mathrm{ng} / \mathrm{ml}) /$ high $(>50 \mathrm{ng} / \mathrm{ml}) / \mathrm{N} / \mathrm{A}$

Ascites, yes / no

Cirrhosis, yes / no

Tumor necrosis, yes / no / N/A
Number of cases $(n=50)$

$47.8 \pm 20.8$

$27 / 23$

$20 / 4 / 15 / 11$

$23 / 7 / 20 / 0$

$9.8 \pm 5.3$

14 / 36

$9 / 41$

$4 / 46$

$10 / 16 / 17 / 7$

$21 / 29$

$4 / 10 / 16 / 20$

$8 / 22 / 6 / 0 / 14$

$33 / 15 / 2$

$3 / 47$

$7 / 43$

$30 / 19 / 1$

$1 \quad$ N/A - data not available.

2 bestimated by average number of visible vessels in histological slides of tumor tissue.

$3 \quad{ }^{\mathrm{c}} \mathrm{Gx}-$ Edmondson-Steiner grade not applicable. 


\section{Table 2 (on next page)}

Correlations between HCC/NT ratios of expression levels or fractions of VEGFA isoforms and tumor clinicopathological properties.

Data presented as Spearman's correlation coefficients ( $p$-value). 


\begin{tabular}{|c|c|c|c|c|c|c|c|}
\hline & \multicolumn{4}{|c|}{ Expression level fold change } & \multicolumn{3}{|c|}{ Isoform fraction fold change } \\
\hline & $\begin{array}{l}\text { VEGFA- } \\
\text { spliced }\end{array}$ & $\begin{array}{c}\text { VEGFA- } \\
189\end{array}$ & $\begin{array}{c}\text { VEGFA- } \\
165\end{array}$ & $\begin{array}{c}\text { VEGFA- } \\
121\end{array}$ & $\begin{array}{c}\text { VEGFA- } \\
189\end{array}$ & $\begin{array}{c}\text { VEGFA- } \\
165\end{array}$ & $\begin{array}{c}\text { VEGFA- } \\
121\end{array}$ \\
\hline Gender & $\begin{array}{l}0,007 \\
\left(\mathrm{NS}^{\mathrm{a}}\right)\end{array}$ & $\begin{array}{c}-0,013 \\
(\mathrm{NS})\end{array}$ & $\begin{array}{c}0,068 \\
(\mathrm{NS})\end{array}$ & $\begin{array}{c}-0,001 \\
(\mathrm{NS})\end{array}$ & $\begin{array}{c}0,024 \\
(\mathrm{NS})\end{array}$ & $\begin{array}{c}0,096 \\
(\mathrm{NS})\end{array}$ & $\begin{array}{l}0,085 \\
(\mathrm{NS})\end{array}$ \\
\hline Age & $\begin{array}{c}-0,037 \\
(\mathrm{NS})\end{array}$ & $\begin{array}{c}-0,078 \\
(\mathrm{NS})\end{array}$ & $\begin{array}{c}-0,030 \\
(\mathrm{NS})\end{array}$ & $\begin{array}{c}0,087 \\
(\mathrm{NS})\end{array}$ & $\begin{array}{c}-0,064 \\
(\mathrm{NS})\end{array}$ & $\begin{array}{c}-0,164 \\
(\mathrm{NS})\end{array}$ & $\begin{array}{c}0,168 \\
(\mathrm{NS})\end{array}$ \\
\hline TNM tumor stage & $\begin{array}{c}0,140 \\
(\mathrm{NS})\end{array}$ & $\begin{array}{c}-0,034 \\
(\mathrm{NS})\end{array}$ & $\begin{array}{c}0,194 \\
(\mathrm{NS})\end{array}$ & $\begin{array}{c}0,207 \\
(\mathrm{NS})\end{array}$ & $\begin{array}{l}-0,297 \\
(0,036)\end{array}$ & $\begin{array}{c}-0,070 \\
(\mathrm{NS})\end{array}$ & $\begin{array}{c}0,238 \\
(\mathrm{NS})\end{array}$ \\
\hline BCLC tumor stage & $\begin{array}{l}0,100 \\
(\mathrm{NS})\end{array}$ & $\begin{array}{c}-0,084 \\
(\mathrm{NS})\end{array}$ & $\begin{array}{l}0,141 \\
(\mathrm{NS})\end{array}$ & $\begin{array}{c}0,229 \\
(\mathrm{NS})\end{array}$ & $\begin{array}{c}-0,328 \\
(0,020)\end{array}$ & $\begin{array}{c}-0,082 \\
(\mathrm{NS})\end{array}$ & $\begin{array}{c}0,286 \\
(0,044)\end{array}$ \\
\hline Primary tumor size & $\begin{array}{c}-0,004 \\
(\mathrm{NS})\end{array}$ & $\begin{array}{c}-0,099 \\
(\mathrm{NS})\end{array}$ & $\begin{array}{c}0,002 \\
(\mathrm{NS})\end{array}$ & $\begin{array}{c}0,053 \\
(\mathrm{NS})\end{array}$ & $\begin{array}{c}-0,154 \\
(\mathrm{NS})\end{array}$ & $\begin{array}{c}0,123 \\
(\mathrm{NS})\end{array}$ & $\begin{array}{c}0,103 \\
(\mathrm{NS})\end{array}$ \\
\hline $\begin{array}{l}\text { Intrahepatic } \\
\text { metastases presence }\end{array}$ & $\begin{array}{l}0,037 \\
(\mathrm{NS})\end{array}$ & $\begin{array}{c}-0,034 \\
(\mathrm{NS})\end{array}$ & $\begin{array}{l}0,151 \\
(\mathrm{NS})\end{array}$ & $\begin{array}{l}0,157 \\
(\mathrm{NS})\end{array}$ & $\begin{array}{c}-0,142 \\
(\mathrm{NS})\end{array}$ & $\begin{array}{l}0,019 \\
(\mathrm{NS})\end{array}$ & $\begin{array}{l}0,161 \\
(\mathrm{NS})\end{array}$ \\
\hline $\begin{array}{l}\text { Lymph node } \\
\text { metastases presence }\end{array}$ & $\begin{array}{l}0,103 \\
(\mathrm{NS})\end{array}$ & $\begin{array}{l}0,121 \\
(\mathrm{NS})\end{array}$ & $\begin{array}{l}0,157 \\
(\mathrm{NS})\end{array}$ & $\begin{array}{l}0,153 \\
(\mathrm{NS})\end{array}$ & $\begin{array}{c}-0,081 \\
(\mathrm{NS})\end{array}$ & $\begin{array}{l}-0,348 \\
(0,013)\end{array}$ & $\begin{array}{l}0,247 \\
(\mathrm{NS})\end{array}$ \\
\hline $\begin{array}{l}\text { Distant metastases } \\
\text { presence }\end{array}$ & $\begin{array}{c}-0,209 \\
(\mathrm{NS})\end{array}$ & $\begin{array}{c}-0,199 \\
(\mathrm{NS})\end{array}$ & $\begin{array}{c}-0,066 \\
(\mathrm{NS})\end{array}$ & $\begin{array}{c}-0,230 \\
(\mathrm{NS})\end{array}$ & $\begin{array}{c}-0,087 \\
(\mathrm{NS})\end{array}$ & $\begin{array}{l}0,020 \\
(\mathrm{NS})\end{array}$ & $\begin{array}{c}-0,153 \\
(\mathrm{NS})\end{array}$ \\
\hline $\begin{array}{l}\text { Tumor capsule } \\
\text { presence }\end{array}$ & $\begin{array}{c}-0,241 \\
(\mathrm{NS})\end{array}$ & $\begin{array}{c}-0,097 \\
(\mathrm{NS})\end{array}$ & $\begin{array}{l}-0,359 \\
(0,018)\end{array}$ & $\begin{array}{l}-0,231 \\
(\mathrm{NS})\end{array}$ & $\begin{array}{l}0,160 \\
(\mathrm{NS})\end{array}$ & $\begin{array}{c}-0,208 \\
(\mathrm{NS})\end{array}$ & $\begin{array}{l}0,020 \\
(\mathrm{NS})\end{array}$ \\
\hline $\begin{array}{l}\text { Invasion into blood } \\
\text { vessels }\end{array}$ & $\begin{array}{l}0,161 \\
(\mathrm{NS})\end{array}$ & $\begin{array}{l}0,060 \\
(\mathrm{NS})\end{array}$ & $\begin{array}{l}0,161 \\
(\mathrm{NS})\end{array}$ & $\begin{array}{l}0,265 \\
(\mathrm{NS})\end{array}$ & $\begin{array}{c}-0,091 \\
(\mathrm{NS})\end{array}$ & $\begin{array}{c}-0,077 \\
(\mathrm{NS})\end{array}$ & $\begin{array}{l}0,204 \\
(\mathrm{NS})\end{array}$ \\
\hline Tumor vascularity & $\begin{array}{l}0,123 \\
(\mathrm{NS})\end{array}$ & $\begin{array}{l}0,309 \\
(\mathrm{NS})\end{array}$ & $\begin{array}{l}0,125 \\
(\mathrm{NS})\end{array}$ & $\begin{array}{l}0,010 \\
(\mathrm{NS})\end{array}$ & $\begin{array}{l}0,161 \\
(\mathrm{NS})\end{array}$ & $\begin{array}{c}-0,142 \\
(\mathrm{NS})\end{array}$ & $\begin{array}{c}-0,212 \\
(\mathrm{NS})\end{array}$ \\
\hline $\begin{array}{l}\text { Edmondson-Steiner } \\
\text { differentiation grade }\end{array}$ & $\begin{array}{l}0,012 \\
(\mathrm{NS})\end{array}$ & $\begin{array}{l}-0,168 \\
(\mathrm{NS})\end{array}$ & $\begin{array}{l}0,064 \\
(\mathrm{NS})\end{array}$ & $\begin{array}{l}0,023 \\
(\mathrm{NS})\end{array}$ & $\begin{array}{l}-\mathbf{0 , 3 9 7} \\
(\mathbf{0 , 0 1 6 )}\end{array}$ & $\begin{array}{l}0,152 \\
(\mathrm{NS})\end{array}$ & $\begin{array}{l}0,084 \\
(\mathrm{NS})\end{array}$ \\
\hline Serum AFP level & $\begin{array}{l}0,050 \\
(\mathrm{NS})\end{array}$ & $\begin{array}{c}-0,261 \\
(\mathrm{NS})\end{array}$ & $\begin{array}{l}0,067 \\
(\mathrm{NS})\end{array}$ & $\begin{array}{l}0,200 \\
(\mathrm{NS})\end{array}$ & $\begin{array}{l}-0,420 \\
(0,003)\end{array}$ & $\begin{array}{l}0,248 \\
(\mathrm{NS})\end{array}$ & $\begin{array}{l}0,219 \\
(\mathrm{NS})\end{array}$ \\
\hline Ascites presence & $\begin{array}{c}0,336 \\
(0,017)\end{array}$ & $\begin{array}{l}0,231 \\
(\mathrm{NS})\end{array}$ & $\begin{array}{c}0,324 \\
(0,022)\end{array}$ & $\begin{array}{c}0,318 \\
(0,024)\end{array}$ & $\begin{array}{l}0,067 \\
(\mathrm{NS})\end{array}$ & $\begin{array}{c}-0,120 \\
(\mathrm{NS})\end{array}$ & $\begin{array}{l}0,108 \\
(\mathrm{NS})\end{array}$ \\
\hline Cirrhosis presence & $\begin{array}{l}0,054 \\
(\mathrm{NS})\end{array}$ & $\begin{array}{c}-0,034 \\
(\mathrm{NS})\end{array}$ & $\begin{array}{l}0,082 \\
(\mathrm{NS})\end{array}$ & $\begin{array}{l}0,230 \\
(\mathrm{NS})\end{array}$ & $\begin{array}{c}-0,094 \\
\text { (NS) }\end{array}$ & $\begin{array}{c}-0,066 \\
(\mathrm{NS})\end{array}$ & $\begin{array}{l}0,162 \\
(\mathrm{NS})\end{array}$ \\
\hline $\begin{array}{l}\text { Tumor necrosis } \\
\text { presence }\end{array}$ & $\begin{array}{l}0,006 \\
(\mathrm{NS})\end{array}$ & $\begin{array}{c}-0,118 \\
(\mathrm{NS})\end{array}$ & $\begin{array}{c}-0,062 \\
(\mathrm{NS})\end{array}$ & $\begin{array}{l}0,021 \\
(\mathrm{NS})\end{array}$ & $\begin{array}{c}-0,018 \\
(\mathrm{NS})\end{array}$ & $\begin{array}{c}0,086 \\
(\mathrm{NS})\end{array}$ & $\begin{array}{c}0,024 \\
(\mathrm{NS})\end{array}$ \\
\hline
\end{tabular}

1 aNS - non-significant correlation. 